\title{
On a Class of Multilinear Operator Equations
}

\author{
J. Janno and L. v. Wolfersdorf
}

\begin{abstract}
By means of contraction principle in a Banach space $E$ with a scale of norms $\|\cdot\|_{\sigma}(\sigma \geq 0)$ existence, uniqueness and stability of solutions are proved for a general class of operator equations $u+G_{0} u+G_{1} u=g$ including multilinear ones where $G_{0}, G_{1} \in(E \rightarrow E)$ are some operators. The theorems are applicable to equations with operators of generalized convolution type.
\end{abstract}

Keywords: Nonlinear operator equations, scale of norms, existence, stability

AMS subject classification: 47 H 15, 45 G 10, 45 D 05

\section{Introduction}

Recently, by Bukhgeim [2] and the authors [6], global existence and stability theorems for some types of abstract one-dimensional nonlinear convolution equations are proved using norms with exponential weights. This method has been applied also to multidimensional nonlinear Volterra equations of convolution type in Lebesgue spaces $L_{p}$ with mixed norm [5].

In the present paper this approach is extended to a general class of nonlinear operator equations in a Banach space with a scale of norms. In particular, this class of operator equations includes some types of equations with multilinear operators. As a special case of such multilinear operators a class of operators of generalized convolution type in classical spaces $C$ and $L_{\infty}$ is dealt with.

\section{Main theorem}

We study the operator equation

$$
u+G_{0} u+G_{1} u=g
$$

in a Banach space $E$, which is endowed with a scale of norms $\|\cdot\|_{\sigma}(\sigma \geq 0)$ satisfying the condition

$$
\psi(\sigma) \cdot\|u\|_{0} \leq\|u\|_{\sigma} \leq\|u\|_{0} \quad(u \in E, \sigma \geq 0)
$$

J. Janno: Estonian Acad. Sci., Institute of Cybernetics, EE - 0026 Tallinn, Estonia. The paper was written during a stay of the author at the Freiberg University of Mining and Technology with a grant of DFG from September 1995 to December 1995.

L. v. Wolfersdorf: Techn. Univ. Bergakademie Freiberg, Fakultät für Mathematik und Informatik, D - 09596 Freiberg 
with a continuous positive function $\psi$. For the operators $G_{0}, G_{1} \in(E \rightarrow E)$ there hold the following assumptions:

(A1) $\left\|G_{0} u_{1}-G_{0} u_{2}\right\|_{\sigma} \leq M_{0}\left(\left\|u_{1}\right\|_{\sigma},\left\|u_{2}\right\|_{\sigma}, \sigma\right) \cdot\left\|u_{1}-u_{2}\right\|_{\sigma} \quad\left(u_{1}, u_{2} \in E, \sigma \geq 0\right)$.

(A2) $\left\|G_{1} u\right\|_{\sigma} \rightarrow 0$ as $\sigma \rightarrow \infty \quad(u \in E)$.

(A3) $\left\|G_{1} u_{1}-G_{1} u_{2}\right\|_{\sigma} \leq M_{1}\left(f,\left\|u_{1}-f\right\|_{\sigma},\left\|u_{2}-f\right\|_{\sigma}, \sigma\right) \cdot\left\|u_{1}-u_{2}\right\|_{\sigma}$ for every $u_{1}, u_{2}, f \in$ $E$ and $\sigma \geq 0$ with $\left\|u_{i}-f\right\|_{\sigma} \leq \rho_{0}(i=1,2)$ where $\rho_{0}$ is some given positive number and the coefficients $M_{0}$ and $M_{1}$ satisfy the following conditions:

(A4) $M_{0} \in C\left(\mathbb{R}_{+}^{3} \rightarrow \mathbb{R}_{+}\right)$

$M_{0}\left(\rho_{1}, \rho_{2}, \sigma\right)$ is increasing in $\rho_{1}, \rho_{2}$ and decreasing in $\sigma$

$\lim _{\sigma \rightarrow \infty} M_{0}\left(\rho_{1}, \rho_{2}, \sigma\right)=0$ for any positive $\rho_{1}, \rho_{2}$.

(A5) ${ }^{\prime} M_{1} \in C\left(E \times\left[0, \rho_{0}\right]^{2} \times \mathbb{R}_{+} \rightarrow \mathbb{R}_{+}\right)$

$M_{1}\left(f, \rho_{1}, \rho_{2}, \sigma\right)$ is increasing in $\rho_{1}, \rho_{2}$ and decreasing in $\sigma$

$m_{1}\left(f, \rho_{1}, \rho_{2}\right):=\lim _{\sigma \rightarrow \infty} M_{1}\left(f, \rho_{1}, \rho_{2}, \sigma\right) \in C\left(E \times\left[0, \rho_{0}\right]^{2} \rightarrow \mathbb{R}_{+}\right)$

$Q(f):=m_{1}(f, 0,0)<1$.

Here, as usual, $\mathbb{R}_{+}$denotes the positive real semi-axis.

Let us first draw some simple conclusions from the conditions (A2), (A4) and (A5). Due to the monotonicity of $M_{0}$ in $\sigma$ and the last condition of (A4), for every pair $\rho>0$ and $\varepsilon>0$ we can define

$$
\sigma_{0}(\rho, \varepsilon)=\inf \left\{\sigma_{*} \in[0, \infty): M_{0}(\rho, \rho, \sigma) \leq \varepsilon \text { if } \sigma \geq \sigma_{*}\right\} .
$$

Moreover, it follows from the condition (A4) that, for any $\varepsilon>0$,

$$
\begin{aligned}
& M_{0}\left(\rho_{1}, \rho_{2}, \sigma\right) \leq \varepsilon \quad \text { if } 0 \leq \rho_{i} \leq \rho(i=1,2) \text { and } \sigma \geq \sigma_{0}(\rho, \varepsilon) \\
& \sigma_{0} \in C\left(\mathbb{R}_{+}^{2} \rightarrow \mathbb{R}_{+}\right) .
\end{aligned}
$$

Let us further denote

$$
q(f)=\frac{2+Q(f)}{3}
$$

Evidently, by (1.5) and the last condition of (A5) we have the inequalities

$$
Q(f)<\frac{1+Q(f)}{2}<q(f)<1 .
$$

Thus, due to the last condition of (A5) and the monotonicity of $M_{1}$ in $\rho_{1}, \rho_{2}$ and $\sigma$, respectively, we can define

$$
r_{1}(f)=\sup \left\{r \in\left(0, \rho_{0}\right]: m_{1}\left(f, \rho_{1}, \rho_{2}\right) \leq \frac{1+Q(f)}{2} \quad \text { if } \rho_{1}, \rho_{2} \leq r\right\}
$$

and

$$
\sigma_{1}(f)=\inf \left\{\sigma_{*} \in[0, \infty): M_{1}\left(f, r_{1}(f), r_{1}(f), \sigma\right) \leq q(f) \text { if } \sigma \geq \sigma_{*}\right\}
$$


Then the first two conditions of (A5) imply

$$
\begin{aligned}
& M\left(f, \rho_{1}, \rho_{2}, \sigma\right) \leq q(f)<1 \text { if } 0 \leq \rho_{1}, \rho_{2} \leq r_{1}(f) \text { and } \sigma \geq \sigma_{1}(f) \\
& q, r_{1}, \sigma_{1} \in C\left(E \rightarrow \mathbb{R}_{+}\right) .
\end{aligned}
$$

Finally, in view of (A2), for every $\rho>0$ and $f \in E$ we can define

$$
\sigma_{2}(f, \rho)=\inf \left\{\sigma_{*} \in[0, \infty):\left\|G_{1} f\right\|_{\sigma} \leq \frac{1-q(f)}{2} \rho \text { if } \sigma \geq \sigma_{*}\right\} .
$$

Then there holds

$$
\left\|G_{1} f\right\|_{\sigma} \leq \frac{1-q(f)}{2} \rho
$$

if $\sigma \geq \sigma_{2}(f, \rho)$ and due to the continuity of $G_{1}$ (see (A3)) and $q$ we have

$$
\sigma_{2} \in C\left(E \times \mathbb{R}_{+} \rightarrow \mathbb{R}_{+}\right) .
$$

Now we can formulate our main result.

Theorem 1. Let (1.2) and the assumptions (A1) - (A5) be satisfied. Then for every $g \in E$ equation (1.1) has a unique solution $u \in E$. For the solutions $u_{1}$ and $u_{2}$ corresponding to data $g_{1}$ and $g_{2}$, respectively, the estimates

(S1) $\left\|u_{1}-u_{2}\right\|_{\sigma} \leq \frac{2}{1-q\left(f_{1}\right)}\left\|g_{1}-g_{2}\right\|_{\sigma}$ if $\sigma \geq \tilde{\sigma}\left(f_{1}, p\left(g_{1}, g_{2}\right)\right)$ and $\left\|g_{1}-g_{2}\right\|_{0} \leq$ $\tilde{\delta}\left(f_{1}, p\left(g_{1}, g_{2}\right)\right)$

$$
\begin{aligned}
& \left\|u_{1}-u_{2}\right\|_{0} \leq \frac{2}{\left(1-q\left(f_{1}\right)\right) \cdot \psi\left(\tilde{\sigma}\left(f_{1}, p\left(g_{1}, g_{2}\right)\right)\right)}\left\|g_{1}-g_{2}\right\|_{0} \text { if }\left\|g_{1}-g_{2}\right\|_{0} \leq \tilde{\delta}\left(f_{1},\right. \\
& \left.p\left(g_{1}, g_{2}\right)\right)
\end{aligned}
$$

hold. Here $p\left(g_{1}, g_{2}\right)=\max _{i=1,2}\left\{2\left\|G_{0} g_{i}\right\|_{0}+\left\|g_{i}\right\|_{0}\right\}, f_{1}$ is the solution of the equation

$$
f+G_{0} f=g
$$

with $g=g_{1}, q$ is defined by (1.5), $\tilde{\sigma}\left(f_{1}, \cdot\right) \geq 0$ and $\tilde{\delta}\left(f_{1}, \cdot\right)>0$ are certain continuous functions.

Proof. Let us define the balls

$$
B_{\rho, \sigma}(v)=\left\{u \in E:\|u-v\|_{\sigma} \leq \rho\right\} \quad(\rho>0, \sigma \geq 0, v \in E)
$$

in $E$.

Step 1. At first we show that the auxiliary equation (1.10) has a solution in the ball $B_{R, \sigma}(g)$, where $R=2\left\|G_{0} g\right\|_{0}$ and $\sigma$ is chosen large enough. By assumption (A1) and (1.2) for the operator $A_{0} f=g-G_{0} f$ we derive the estimates

$$
\begin{aligned}
\left\|A_{0} f-g\right\|_{\sigma}=\left\|G_{0} f\right\|_{\sigma} & \leq\left\|G_{0} f-G_{0} g\right\|_{\sigma}+\left\|G_{0} g\right\|_{\sigma} \\
& \leq M_{0}\left(\|f\|_{\sigma},\|g\|_{\sigma}, \sigma\right) \cdot\|f-g\|_{\sigma}+\frac{R}{2}
\end{aligned}
$$


and

$$
\left\|A_{0} f_{1}-A_{0} f_{2}\right\|_{\sigma}=\left\|G_{0} f_{1}-G_{0} f_{2}\right\|_{\sigma} \leq M_{0}\left(\left\|f_{1}\right\|_{\sigma},\left\|f_{2}\right\|_{\sigma}, \sigma\right) \cdot\left\|f_{1}-f_{2}\right\|_{\sigma} .
$$

If $f_{1}, f_{2}, f \in B_{R, \sigma}(g)$, then

$$
\|f\|_{\sigma} \leq R+\|g\|_{0} \quad \text { and } \quad\left\|f_{i}\right\|_{\sigma} \leq R+\|g\|_{0} \quad(i=1,2) .
$$

Now by (1.3) we have

$$
\left\|A_{0} f-g\right\|_{\sigma} \leq \frac{1}{2} R+\frac{R}{2}=R \quad \text { and } \quad\left\|A_{0} f_{1}-A_{0} f_{2}\right\|_{\sigma} \leq \frac{1}{2}\left\|f_{1}-f_{2}\right\|_{\sigma}
$$

if $f, f_{i} \in B_{R ; \sigma}(g)$ with $\sigma \geq \sigma_{0}\left(R+\|g\|_{0}, \frac{1}{2}\right)$. Thus, for such $\sigma$ the operator $A_{0}$ maps $B_{R, \sigma}(g)$ into itself and is a contraction in $B_{R, \sigma}(g)$. This implies the existence of a solution to equation (1.10) in $B_{R, \sigma}(g)$, where $R=2\left\|G_{0} g\right\|_{0}$ and $\sigma \geq \sigma_{0}\left(R+\|g\|_{0}, \frac{1}{2}\right)$.

Step 2. Next we are going to show that a unique solution of equation (1.1) exists in the ball $B_{\rho, \sigma}(f)$, where $\rho$ is small enough, $\sigma$ is large enough and $f$ is a solution to equation (1.10). Let us denote

$$
A u=g-G_{0} u-G_{1} u
$$

By virtue of (1.10) and the assumptions (A1) and (A3) we derive the estimates

$$
\begin{aligned}
\| A u & -f \|_{\sigma} \\
& =\left\|G_{0} f-G_{0} u-G_{1} u+G_{1} f-G_{1} f\right\|_{\sigma} \\
& \leq\left\|G_{0} f-G_{0} u\right\|_{\sigma}+\left\|G_{1} f-G_{1} u\right\|_{\sigma}+\left\|G_{1} f\right\|_{\sigma} \\
& \leq\left[M_{0}\left(\|f\|_{\sigma},\|u\|_{\sigma}, \sigma\right)+M_{1}\left(f, 0,\|u-f\|_{\sigma}, \sigma\right)\right] \cdot\|u-f\|_{\sigma}+\left\|G_{1} f\right\|_{\sigma}
\end{aligned}
$$

if $\|u-f\|_{\sigma} \leq \rho_{0}$ and

$$
\begin{aligned}
\| A u_{1} & -A u_{2} \|_{\sigma} \\
& \leq\left\|G_{0} u_{1}-G_{0} u_{2}\right\|_{\sigma}+\left\|G_{1} u_{1}-G_{1} u_{2}\right\|_{\sigma} \\
& \leq\left[M_{0}\left(\left\|u_{1}\right\|_{\sigma},\left\|u_{2}\right\|_{\sigma}, \sigma\right)+M_{1}\left(f,\left\|u_{1}-f\right\|_{\sigma},\left\|u_{2}-f\right\|_{\sigma}, \sigma\right)\right] \cdot\left\|u_{1}-u_{2}\right\|_{\sigma}
\end{aligned}
$$

if $\left\|u_{i}-f\right\|_{\sigma} \leq \rho_{0} \quad(i=1,2)$. We further estimate the coefficients $M_{0}$ and $M_{1}$ in (1.11) and (1.12). Suppose that $u, u_{1}, u_{2} \in B_{\rho, \sigma}(f)$, where $\rho \leq r_{1}(f)$. Then we have

$$
\|u\|_{\sigma} \leq r_{1}(f)+\|f\|_{0} \cdot \text { and } \quad\left\|u_{i}\right\|_{\sigma} \leq r_{1}(f)+\|f\|_{0} \quad(i=1,2)
$$

and (1.3), (1.6) imply

$$
M_{0}\left(\|f\|_{\sigma},\|u\|_{\sigma}, \sigma\right) \leq \frac{1-q(f)}{2} \text { and } M_{0}\left(\left\|u_{1}\right\|_{\sigma},\left\|u_{2}\right\|_{\sigma}, \sigma\right) \leq \frac{1-q(f)}{2}
$$


if $\sigma \geq \sigma_{0}\left(r_{1}(f)+\|f\|_{0}, \frac{1-q(f)}{2}\right)$ and

$$
M_{1}\left(f, 0,\|u-f\|_{\sigma}, \sigma\right) \leq q(f) \text { and } M_{1}\left(f,\left\|u_{1}-f\right\|_{\sigma},\left\|u_{2}-f\right\|_{\sigma}, \sigma\right) \leq q(f)
$$

if $\sigma \geq \sigma_{1}(f)$. Combining (1.11), (1.12) with (1.14), (1.15) and also taking (1.8) into account, we obtain

$$
\|A u-f\|_{\sigma} \leq\left[\frac{1-q(f)}{2}+q(f)\right] \rho+\frac{1-q(f)}{2} \rho=\rho
$$

and

$$
\left\|A u_{1}-A u_{2}\right\|_{\sigma} \leq\left[\frac{1-q(f)}{2}+q(f)\right] \cdot\left\|u_{1}-u_{2}\right\|_{\sigma}=\frac{1+q(f)}{2} \cdot\left\|u_{1}-u_{2}\right\|_{\sigma}
$$

if $u, u_{1}, u_{2} \in B_{\rho, \sigma}(f), \rho \leq r_{1}(f)$ and $\sigma \geq \sigma_{3}(f, \rho)$ where

$$
\sigma_{3}(f, \rho)=\max \left\{\sigma_{1}(f), \sigma_{0}\left(r_{1}(f)+\|f\|_{0}, \frac{1-q(f)}{2}, \sigma_{2}(f, \rho)\right\} .\right.
$$

Since $\frac{1+q(f)}{2}<1$, we have that $A$ maps $B_{\rho, \sigma}(f)$ into itself and is a contraction in $B_{\rho, \sigma}(f)$ if $\rho \leq r_{1}(f)$ and $\sigma \geq \sigma_{3}(f, \rho)$. Thus, equation (1.1) has a unique solution $u$ in every ball $B_{\rho, \sigma}(f)$, where $\rho \leq r_{1}(f)$ and $\sigma \geq \sigma_{3}(f, \rho)$. Particularly, this proves the existence result of Theorem 1.

Step 3. Let us prove the uniqueness of the solution of equation (1.1) in $E$. Suppose that $u_{1} \in E$ and $u_{2} \in E$ are two arbitrary solutions of equation (1.1). Then

$$
\begin{aligned}
\left\|u_{i}-f\right\|_{\sigma} & =\left\|G_{0} f-G_{0} u_{i}-G_{1} u_{i}\right\|_{\sigma} \\
& \leq\left\|G_{0} f-G_{0} u_{i}\right\|_{\sigma}+\left\|G_{1} u_{i}\right\|_{\sigma} \\
& \leq M_{0}\left(\|f\|_{\sigma},\left\|u_{i}\right\|_{\sigma}, \sigma\right) \cdot\left\|u_{i}-f\right\|_{\sigma}+\left\|G_{1} u_{i}\right\|_{\sigma} \quad(i=1,2) .
\end{aligned}
$$

Now it follows from (1.2) and the assumptions (A4), (A2) that $\left\|u_{i}-f\right\|_{\sigma} \leq r_{1}(f)$ if $\sigma$ is greater than some number $\sigma_{4}$ which depends on $u_{1}, u_{2}$ and $f$. Thus, $u_{i} \in B_{r_{1}(f), \sigma}(f)$ if $\sigma \geq \sigma_{4}$. Taking $\sigma \geq \max \left\{\sigma_{4}, \sigma_{3}\left(f, r_{1}(f)\right)\right\}$, the solutions $u_{1}$ and $u_{2}$ belong to a ball where the uniqueness of the solution has already been shown. Thus, $u_{1}=u_{2}$.

Step 4. Now we derive a stability estimate for the solution of the auxiliary equation (1.10), which is uniquely determined as we have just shown. Suppose that $f_{1}$ and $f_{2}$ are the solutions of (1.10) with $g$ replaced by $g_{1}$ and $g_{2}$, respectively. Then by assumption (A1)

$$
\begin{aligned}
\left\|f_{1}-f_{2}\right\|_{\sigma} & \leq\left\|g_{1}-g_{2}\right\|_{\sigma}+\left\|G_{0} f_{1}-G_{0} f_{2}\right\|_{\sigma} . \\
& \leq\left\|g_{1}-g_{2}\right\|_{\sigma}+M_{0}\left(\left\|f_{1}\right\|_{\sigma},\left\|f_{2}\right\|_{\sigma}, \sigma\right) \cdot\left\|f_{1}-f_{2}\right\|_{\sigma} .
\end{aligned}
$$

From Step 1 it follows that $\left\|f_{i}-g_{i}\right\|_{\sigma} \leq R_{i}$ if $\sigma \geq \sigma_{0}\left(R_{i}+\left\|g_{i}\right\|_{0}, \frac{1}{2}\right)$, where $R_{i}=2\left\|G_{0} g_{i}\right\|_{0}$. Thus, observing (1.2) we have

$$
\left\|f_{i}\right\|_{o} \leq 2\left\|G_{0} g_{i}\right\|_{0}+\left\|g_{i}\right\|_{0} \leq \max _{j=1,2}\left\{2\left\|G_{0} g_{j}\right\|_{0}+\left\|g_{j}\right\|_{0}\right\}=p\left(g_{1}, g_{2}\right)
$$


if $\sigma \geq \sigma_{0}\left(p\left(g_{1}, g_{2}\right), \frac{1}{2}\right)$. In view of (1.18) condition (1.3) implies

$$
M_{0}\left(\left\|f_{1}\right\|_{\sigma},\left\|f_{2}\right\|_{\sigma}, \sigma\right) \leq \frac{1}{2} \quad \text { if } \sigma \geq \sigma_{0}\left(p\left(g_{1}, g_{2}\right), \frac{1}{2}\right) .
$$

This together with (1.17) yields

$$
\left\|f_{1}-f_{2}\right\|_{\sigma} \leq 2\left\|g_{1}-g_{2}\right\|_{\sigma} \quad \text { if } \sigma \geq \sigma_{0}\left(p\left(g_{1}, g_{2}\right), \frac{1}{2}\right) \text {. }
$$

Step 5. Finally, let us derive the estimates (S1) and (S2). Suppose that $u_{1}$ and $u_{2}$ are the solutions of equation (1.1) with $g$ replaced by $g_{1}$ and $g_{2}$, respectively. Then, by the assumptions (A1) and (A3),

$$
\begin{aligned}
\left\|u_{1}-u_{2}\right\|_{\sigma} \leq & \left\|G_{0} u_{1}-G_{0} u_{2}\right\|_{\sigma}+\left\|G_{1} u_{1}-G_{1} u_{2}\right\|_{\sigma}+\left\|g_{1}-g_{2}\right\|_{\sigma} \\
\leq & {\left[M_{0}\left(\left\|u_{1}\right\|_{\sigma},\left\|u_{2}\right\|_{\sigma}, \sigma\right)+M_{1}\left(f_{1},\left\|u_{1}-f_{1}\right\|_{\sigma},\left\|u_{2}-f_{1}\right\|_{\sigma}, \sigma\right)\right] } \\
& \times\left\|u_{1}-u_{2}\right\|_{\sigma}+\left\|g_{1}-g_{2}\right\|_{\sigma}
\end{aligned}
$$

if $\left\|u_{i}-f_{1}\right\|_{\sigma} \leq \rho_{0}(i=1,2)$ where as above $f_{1}$ is the solution of equation (1.10) for $g=g_{1}$. We estimate the quantities $\left\|u_{1}\right\|_{\sigma},\left\|u_{2}\right\|_{\sigma}$ and $\left\|u_{1}-f_{1}\right\|_{\sigma},\left\|u_{2}-f_{1}\right\|_{\sigma}$ in (1.20). It follows from Step 2 that

$$
\left\|u_{1}-f_{1}\right\|_{\sigma} \leq r_{1}\left(f_{1}\right) \quad \text { if } \sigma \geq \sigma_{3}\left(f_{1}, r_{1}\left(f_{1}\right)\right)
$$

and

$$
\left\|u_{2}-f_{2}\right\|_{\sigma} \leq \frac{1}{2} r_{1}\left(f_{2}\right) \quad \text { if } \sigma \geq \sigma_{3}\left(f_{2}, \frac{1}{2} r_{1}\left(f_{2}\right)\right) .
$$

By virtue of the continuity properties (1.7), (1.4) and (1.9) the functional $\sigma_{3}$ defined by (1.16) is also continuous in its arguments. Thus, there exists $\delta\left(f_{1}\right) \in\left(0, \frac{1}{2} r_{1}\left(f_{1}\right)\right)$ such that

$$
\sigma_{3}\left(f_{2}, \frac{1}{2} r_{1}\left(f_{2}\right)\right) \leq 2 \sigma_{3}\left(f_{1}, \frac{1}{2} r_{1}\left(f_{1}\right)\right) \quad \text { and } \quad \frac{1}{2} r_{2}\left(f_{2}\right) \leq r_{1}\left(f_{1}\right)-2 \delta\left(f_{1}\right)
$$

if $\left\|f_{1}-f_{2}\right\|_{0} \leq 2 \delta\left(f_{1}\right)$. From (1.22) and (1.2) we now obtain

$$
\left\|u_{2}-f_{1}\right\|_{\sigma} \leq\left\|u_{2}-f_{2}\right\|_{\sigma}+\left\|f_{2}-f_{1}\right\|_{0} \leq r_{1}\left(f_{1}\right)
$$

if $\sigma \geq 2 \sigma_{3}\left(f_{1}, \frac{1}{2} r_{1}\left(f_{1}\right)\right)$ and $\left\|f_{1}-f_{2}\right\|_{0} \leq 2 \delta\left(f_{1}\right)$. Denote

$$
\sigma_{5}\left(f_{1}\right)=\max \left\{\sigma_{3}\left(f_{1}, r_{1}\left(f_{1}\right)\right), 2 \sigma_{3}\left(f_{1}, \frac{1}{2} r_{1}\left(f_{1}\right)\right)\right\}
$$

The estimates (1.21) and (1.23) imply

$$
\left\|u_{i}\right\|_{\sigma} \leq\left\|f_{1}\right\|_{0}+r_{1}\left(f_{1}\right) \quad(i=1,2) .
$$

if $\sigma \geq \sigma_{5}\left(f_{1}\right)$ and $\left\|f_{1}-f_{2}\right\|_{0} \leq 2 \delta\left(f_{1}\right)$. With the help of the bounds (1.21) and (1.23) for $\left\|u_{i}-f_{1}\right\|_{\sigma}$ and (1.24) for $\left\|u_{i}\right\|_{\sigma}$ and the conditions (1.3) and (1.6) we continue the estimation of $\left\|u_{1}-u_{2}\right\|_{\sigma}$ in (1.20) obtaining

$$
\left\|u_{1}-u_{2}\right\|_{\sigma} \leq \frac{1+q\left(f_{1}\right)}{2} \cdot\left\|u_{1}-u_{2}\right\|_{\sigma}+\left\|g_{1}-g_{2}\right\|_{\sigma}
$$




$$
\sigma \geq \sigma_{5}\left(f_{1}\right), \sigma \geq \sigma_{0}\left(\left\|f_{1}\right\|_{0}+r_{1}\left(f_{1}\right), \frac{1-q\left(f_{1}\right)}{2}\right), \sigma \geq \sigma_{1}\left(f_{1}\right),\left\|f_{1}-f_{2}\right\|_{0} \leq 2 \delta\left(f_{1}\right) .
$$

Since

$$
\sigma_{5}\left(f_{1}\right) \geq \sigma_{0}\left(\|f\|_{0}+r_{1}\left(f_{1}\right), \frac{1-q\left(f_{1}\right)}{2}\right) \quad \text { and } \quad \sigma_{5}\left(f_{1}\right) \geq \sigma_{1}\left(f_{1}\right)
$$

we obtain

$$
\left\|u_{1}-u_{2}\right\|_{\sigma} \leq \frac{2}{1-q\left(f_{1}\right)} \cdot\left\|g_{1}-g_{2}\right\|_{\sigma}
$$

if $\sigma \geq \sigma_{5}\left(f_{1}\right)$ and $\left\|f_{1}-f_{2}\right\|_{0} \leq 2 \delta\left(f_{1}\right)$. Taking (1.19) and (1.2) into account we see that (1.25) holds if

$$
\sigma \geq \tilde{\sigma}\left(f_{1}, p\left(g_{1}, g_{2}\right)\right)=\max \left\{\sigma_{5}\left(f_{1}\right) ; \sigma_{0}\left(p\left(g_{1}, g_{2}\right), \frac{1}{2}\right)\right\}
$$

and

$$
\left\|g_{1}-g_{2}\right\|_{0} \leq \tilde{\delta}\left(f_{1}, p\left(g_{1}, g_{2}\right)\right)=\delta\left(f_{1}\right) \cdot \psi\left(\sigma_{0}\left(p\left(q_{1}, g_{2}\right), \frac{1}{2}\right)\right) .
$$

Thus, we have proved the estimate (S1). But the estimate (S1) together with (1.2) implies the estimate (S2). Finally, since $\sigma_{0}$ is continuous, $\psi>0$ and $\delta>0$, the functions $\tilde{\sigma}\left(f_{1}, \cdot\right)$ and $\tilde{\delta}\left(f_{1}, \cdot\right)$ in the estimates (S1) and (S2) are also continuous and $\tilde{\delta}>0$. The proof is complete

\section{Equation with multilinear operator}

As a particular case of equation (1.1) we consider the operator equation

$$
u+G_{0} u+\sum_{k=2}^{N} \sum_{j=1}^{n_{k}} K_{k, j}\left[G_{k, 1}^{j} u, \ldots, G_{k, k}^{j} u\right]=g
$$

where $N \geq 2$ and $n_{k} \geq 1, G_{k, i}^{j} \in\left(E \rightarrow E_{k, i}^{j}\right), E_{k, i}^{j}(1 \leq i \leq k)$ are Banach spaces and $K_{k, j}$ are multilinear operators from $E_{k, 1}^{j} \times \ldots \times E_{k, k}^{j}$ into $E$. We suppose that the spaces $E_{k, i}^{j}$ are endowed with scales of norms $\|\cdot\|_{k, i, j, \sigma}(\sigma \geq 0)$ which satisfy the condition

$$
\|u\|_{k, i, j, \sigma} \leq\|u\|_{k, i, j, 0} \quad\left(u \in E_{k, i}^{j}, \sigma \geq 0\right)
$$

and for the operators $K_{k, j}$ and $G_{k, i}^{j}$ there hold the following assumptions:

(B1) $\left\|K_{k, j}\left[f_{1}, \ldots, f_{k}\right]\right\|_{\sigma} \leq c_{k, j} \prod_{i=1}^{k}\left\|f_{i}\right\|_{k, i, j, \sigma}$ for $f_{i} \in E_{k, i}^{j}\left(1 \leq i \leq k, \sigma \geq 0, c_{k, j} \geq 0\right)$.

(B2) $\left\|K_{k, j}\left[f_{1}, \ldots, f_{k}\right]\right\|_{\sigma} \leq \lambda_{k, j}(\sigma) \cdot \prod_{l \neq i}\left\|f_{l}\right\|_{k, l, j, \sigma} \cdot\left\|f_{i}\right\|_{k, i, j, 0}$ for $f_{l} \in E_{k, l}^{j}(1 \leq l \leq$ $k, \sigma \geq 0, i=1, \ldots, k)$. 
(B3) $\left\|G_{k, i}^{j} u_{1}-G_{k, i}^{j} u_{2}\right\|_{k, i, j, \sigma} \leq M_{k, i}^{j}\left(\left\|u_{1}\right\|_{\sigma},\left\|u_{2}\right\|_{\sigma}\right) \cdot\left\|u_{1}-u_{2}\right\|_{\sigma}$ for $u_{1}, u_{2} \in E(\sigma \geq 0)$ where the coefficients $\lambda_{k, j}$ and $M_{k, i}^{j}$ satisfy the following conditions:

(B4) $\lambda_{k, j} \in C\left(\mathbb{R}_{+} \rightarrow \mathbb{R}_{+}\right), \lambda_{k, j}^{i}$ is decreasing, $\lim _{\sigma \rightarrow \infty} \lambda_{k, j}^{i}(\sigma)=0$.

(B5) $M_{k, i}^{j} \in C\left(\mathbb{R}_{+}^{2} \rightarrow \mathbb{R}_{+}\right), M_{k, i}^{j}\left(\rho_{1}, \rho_{2}\right)$ is increasing in $\rho_{1}$ and $\rho_{2}$.

Concerning the operator $G_{0}$ we assume (A1) and (A4).

Theorem 2. Let (1.2) and (2.2), the assumptions (B1) - (B5) as well as the assumptions (A1) and (A4) be satisfied. Then equation (2.1) has for every.g $\in E$ a unique solution $u \in E$. For the solutions $u_{1}$ and $u_{2}$ corresponding to data $g_{1}$ and $g_{2}$, respectively, the estimates

(C1) $\left\|u_{1}-u_{2}\right\|_{\sigma} \leq 6\left\|g_{1}-g_{2}\right\|_{\sigma}$ if $\sigma \geq \tilde{\sigma}\left(f_{1}, p\left(g_{1}, g_{2}\right)\right)$ and $\left\|g_{1}-g_{2}\right\|_{0} \leq \tilde{\delta}\left(f_{1}, p\left(g_{1}, g_{2}\right)\right)$

(C2) $\left\|u_{1}-u_{2}\right\|_{0} \leq \frac{6}{\psi\left(\tilde{\sigma}\left(f_{1}, p\left(g_{1}, g_{2}\right)\right)\right)}\left\|g_{1}-g_{2}\right\|_{0}$. if $\left\|g_{1}-g_{2}\right\|_{0} \leq \tilde{\delta}\left(f_{1}, p\left(g_{1}, g_{2}\right)\right)$

hold. Here $p\left(g_{1}, g_{2}\right)$ is defined as in Theorem $1, \tilde{\sigma}\left(f_{1}, \cdot\right) \geq 0$ and $\tilde{\delta}\left(f_{1}, \cdot\right)>0$ are certain continuous functions.

Proof. Theorem 2 reduces to Theorem 1 if the operator

$$
G_{1} u=\sum_{k=2}^{N} \sum_{j=1}^{n_{k}} K_{k, j}\left[G_{k, 1}^{j} u, \ldots, G_{k, k}^{j} u\right]
$$

satisfies the conditions (A2), (A3) and (A5) with $Q(f)=0$.

The assumptions (B2) and (2.2) imply

$$
\left\|K_{k, j}\left[f_{1}, \ldots, f_{k}\right]\right\|_{\sigma} \leq \lambda_{k, j}(\sigma) \cdot \prod_{l \neq i}\left\|f_{l}\right\|_{k, l, j, p_{l}} \cdot\left\|f_{i}\right\|_{k, i, j, 0}
$$

for $f_{l} \in E_{k, l}^{j}$ and $p_{l} \in\{0, \sigma\}(1 \leq l \leq k, \sigma \geq 0, i=1, \ldots, k)$. Condition (A2) is a simple consequence of assumption (B4) and $(2.4)$ with $\left(p_{1}, \ldots, p_{k}\right)=(0, \ldots, 0)$.

Let us show condition (A3). Due to the multilinearity of $K_{k, j}$ we can write

$$
\begin{aligned}
K_{k, j}\left[G_{k, 1}^{j} u_{1}, \ldots, G_{k, k}^{j} u_{1}\right]-K_{k, j}\left[G_{k, 1}^{j} u_{2}, \ldots, G_{k, k}^{j} u_{2}\right] \\
=\sum_{p=1}^{k} \sum_{l_{p}^{\prime}} K_{k, j}\left[\chi_{1}^{l}, \ldots, \chi_{p-1}^{l}, G_{k, p}^{j} u_{1}-G_{k, p}^{j} u_{2}, \chi_{p+1}^{l}, \ldots, \chi_{k}^{l}\right]
\end{aligned}
$$

where $l_{p}^{\prime}=\left(l_{1}, \ldots, l_{p-1}, l_{p+1}, \ldots, l_{k}\right)$ with all $l_{s} \in\{0 ; 1\}$

$$
\chi_{s}^{l}= \begin{cases}G_{k, s}^{j} u_{2}-G_{k, s}^{j} f & \text { if } l_{s}=1 \text { and } s \leq p-1 \\ G_{k, s}^{j} u_{1}-G_{k, s}^{j} f & \text { if } l_{s}=1 \text { and } s \geq p+1 \\ G_{k, s}^{j} f & \text { if } l_{s}=0\end{cases}
$$


and $u_{1}, u_{2}$ and $f$ are arbitrary elements in $E$. Taking into account the assumptions (B1) - (B3), we can estimate as follows:

$$
\begin{aligned}
\| K_{k, j}[ & \left.G_{k, 1}^{j} u_{1}, \ldots, G_{k, k}^{j} u_{1}\right]-K_{k, j}\left[G_{k, 1}^{j} u_{2}, \ldots, G_{k, k}^{j} u_{2}\right] \|_{\sigma} \\
\leq & \sum_{p=1}^{k}\left\{c_{k, j} \cdot M_{k, 1}^{j}\left(\left\|u_{2}\right\|_{\sigma},\|f\|_{\sigma}\right) \cdots M_{k, p-1}^{j}\left(\left\|u_{2}\right\|_{\sigma},\|f\|_{\sigma}\right)\right. \\
& \times\left\|u_{2}-f\right\|_{\sigma}^{p-1} \cdot M_{k, p}^{j}\left(\left\|u_{1}\right\|_{\sigma},\left\|u_{2}\right\|_{\sigma}\right) \cdot\left\|u_{1}-u_{2}\right\|_{\sigma} \\
& \times M_{k, p+1}^{j}\left(\left\|u_{1}\right\|_{\sigma},\|f\|_{\sigma}\right) \cdots M_{k, k}^{j}\left(\left\|u_{1}\right\|_{\sigma},\|f\|_{\sigma}\right) \cdot\left\|u_{1}-f\right\|_{\sigma}^{k-p} \\
& +\sum_{l_{p}^{\prime} \neq(1, \ldots, 1)} \lambda_{k, j}(\sigma) \cdot \mu_{1}^{l} \cdots \mu_{p-1}^{l} \\
& \left.\times M_{k, p}^{j}\left(\left\|u_{1}\right\|_{\sigma},\left\|u_{2}\right\|_{\sigma}\right) \cdot\left\|u_{1}-u_{2}\right\|_{\sigma} \cdot \mu_{p+1}^{l} \cdots \mu_{k}^{l}\right\}
\end{aligned}
$$

for $u_{i}, f \in E(i=1,2)$ and $\sigma \geq 0$, where $l_{p}^{\prime}=\left(l_{1}, \ldots, l_{p-1}, l_{p+1}, \ldots, l_{k}\right)$ with all $l_{s} \in\{0 ; 1\}$ and

$$
\mu_{s}^{l}= \begin{cases}M_{k, s}^{j}\left(\left\|u_{2}\right\|_{\sigma},\|f\|_{\sigma}\right) \cdot\left\|u_{2}-f\right\|_{\sigma} \cdot & \text { if } l_{s}=1 \text { and } s \leq p-1 \\ M_{k, s}^{j}\left(\left\|u_{1}\right\|_{\sigma},\|f\|_{\sigma}\right) \cdot\left\|u_{1}-f\right\|_{\sigma} & \text { if } l_{s}=1 \text { and } s \geq p+1 \\ \left\|G_{k, s}^{j} f\right\|_{k, s, j, 0} & \text { if } l_{s}=0 .\end{cases}
$$

Estimating further, we have

$$
\begin{aligned}
& \left\|K_{k, j}\left[G_{k, 1}^{j} u_{1}, \ldots, G_{k, k}^{j} u_{1}\right]-K_{k, j}\left[G_{k, 1}^{j} u_{2}, \ldots, G_{k, k}^{j} u_{2}\right]\right\|_{\sigma} \\
& \leq \bar{M}_{k, j}\left(\left\|u_{1}\right\|_{\sigma},\left\|u_{2}\right\|_{\sigma},\|f\|_{\sigma},\left\|G_{k, 1}^{j} f\right\|_{k, 1, j, 0}, \ldots,\left\|G_{k, k}^{j} f\right\|_{k, k, j, 0}\right)
\end{aligned}
$$

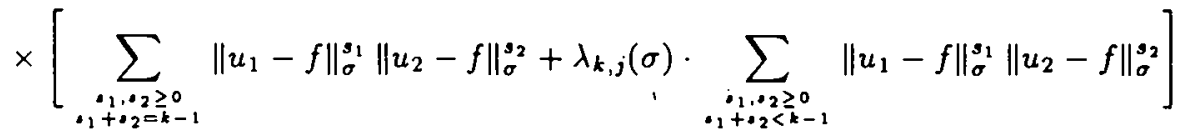

$$
\begin{aligned}
& \times\left\|u_{1}-u_{2}\right\|_{\sigma}
\end{aligned}
$$

for $u_{i}, f \in E$ and $\sigma \geq 0$ where due to assumption (B5) the function $\bar{M}_{k, j}$ is continuous and increasing in each of its arguments. Let us replace the arguments $\left\|u_{1}\right\|_{\sigma},\left\|u_{2}\right\|_{\sigma}$ and $\|f\|_{\sigma}$ of $\bar{M}_{k, j}$ by their majorants $\left\|u_{1}-f\right\|_{\sigma}+\|f\|_{0},\left\|u_{2}-f\right\|_{\sigma}+\|f\|_{0}$ and $\|f\|_{0}$, respectively, and take a sum over $k, j$ to get an estimate for the operator $G_{1}$ defined by (2.3). We obtain

$$
\begin{aligned}
& \left\|G_{1} u_{1}-G_{1} u_{2}\right\|_{\sigma} \leq \bar{M}_{1}\left(f,\left\|u_{1}-f\right\|_{\sigma},\left\|u_{2}-f\right\|_{\sigma}\right) \\
& \times\left[\sum_{\substack{0_{1}, \alpha_{2} \geq 0 \\
1 \leq \alpha_{1}+0_{2} \leq N-1}}\left\|u_{1}-f\right\|_{\sigma}^{s_{1}} \cdot\left\|u_{2}-f\right\|_{\sigma}^{s_{2}}\right. \\
& \left.+\lambda(\sigma) \sum_{\substack{\alpha_{1}, \alpha_{2} \geq 0 \\
, 1+\sigma_{2}<N-1}}\left\|u_{1}-f\right\|_{\sigma}^{s_{1}} \cdot\left\|u_{2}^{\vdots}-f\right\|_{\sigma}^{s_{2}}\right] \cdot\left\|u_{1}-u_{2}\right\|_{\sigma}
\end{aligned}
$$


for $u_{i}, f \in E$ and $\sigma \geq 0$ where due to the mentioned properties of $\bar{M}_{k, j}$, the continuity of $G_{k, i}^{j}$ and assumption (B4) the coefficients $\bar{M}_{1}$ and $\lambda$ satisfy the conditions

$$
\bar{M}_{1} \in C\left(E \times \mathbb{R}_{+}^{2} \rightarrow \mathbb{R}_{+}\right) \text {and } \bar{M}_{1}\left(f, \rho_{1}, \rho_{2}\right) \text { is increasing in } \rho_{1}, \rho_{2}
$$

and

$$
\lambda \in C\left(\mathbb{R}_{+} \rightarrow \mathbb{R}_{+}\right), \quad \lambda \text { is decreasing, } \quad \lim _{\sigma \rightarrow \infty} \lambda(\sigma)=0 .
$$

Hence there follow the assumptions (A3) and (A5) with $Q(f)=0$ and an arbitrary $\rho_{0}$. The Theorem is proved

\section{Equations with generalized convolution operators}

As an example for a multilinear operator we deal with the following integral operator of generalized convolution type:

$$
K\left[f_{1}, \ldots, f_{k}\right](x)=\int_{0}^{x_{n}} \ldots \int_{0}^{x_{1}} m(x, y) \prod_{i=1}^{k} f_{i}\left(\alpha_{i} x-\beta_{i} y\right) d y_{1} \cdots d y_{n}
$$

where

$$
x=\left(x_{1}, \ldots, x_{n}\right), y=\left(y_{1}, \ldots, y_{n}\right) \in D=\prod_{j=1}^{n}\left(0, X_{j}\right) \quad\left(0<X_{j}<\infty\right) .
$$

We consider the operator $K$ in the spaces $E=C(\bar{D})$ and $E=L_{\infty}(D)$ (for more general spaces $L_{p}$ cp. [1]). The function $m$ should have the form

$$
m(x, y)=m_{0}(x, y) \prod_{i=1}^{k} m_{i}\left(\alpha_{i} x-\beta_{i} y\right)
$$

with $m_{0} \in C(\bar{D} \times \bar{D})$ or $m_{0} \in L_{\infty}(D \times D)$, respectively. The parameters

$$
\begin{array}{rll}
\alpha_{i}=\left(\alpha_{i}^{1}, \ldots, \alpha_{i}^{n}\right) & \text { and } & \beta_{i}=\left(\beta_{i}^{1}, \ldots, \beta_{i}^{n}\right) \\
\alpha_{i} x=\left(\alpha_{i}^{1} x_{1}, \ldots, \alpha_{i}^{n} x_{n}\right) & \text { and } & \beta_{i} y=\left(\beta_{i}^{1} y_{1}, \ldots, \beta_{i}^{n} y_{n}\right)
\end{array}
$$

are in $\mathbb{R}^{n}$ where we suppose the componentwise inequalities

$$
0<\beta_{i} \leq \alpha_{i} \quad \text { or } \quad \alpha_{i}-1 \leq \beta_{i}<0 \leq \alpha_{i} \quad(1 \leq i \leq k)
$$

and

$$
\sum_{i=1}^{k} \alpha_{i} \leq 1 \quad \text { and } \quad \sum_{i=1}^{k} \beta_{i} \geq 0
$$

so that $0 \leq \alpha_{i} x-\beta_{2} y \leq X$ if $0 \leq y \leq x \leq X, X=\left(X_{1}, \ldots, X_{n}\right)$. 
The operator $K$ is defined on $F=\prod_{i=1}^{k} E_{i}, E_{i}=L_{p_{i}}(D)\left(1 \leq p_{i} \leq \infty\right)$, and we assume that $m_{i} \in L_{q_{i}}(D)\left(1 \leq q_{i}<\infty\right)$ where

$$
\sum_{i=1}^{k} \frac{1}{r_{i}}=1 \quad \text { and } \quad \frac{1}{r_{i}}=\frac{1}{p_{i}}+\frac{1}{q_{i}}
$$

with $r_{i}<\infty$. Due to these assumptions we have $K \in(F \rightarrow E)$ in both cases $E=C(\bar{D})$ and $E=L_{\infty}(D)$; for the proof in case $E=C(\bar{D})$ compare [1: Section 10/Theorem 1].

We have to show that the operator $K$ fulfils the assumptions (B1) and (B2) with (B4) in suitably chosen scales of norms in $E$ and $F$. For this purpose we use the well-known norms with exponential weights

$$
\|u\|_{\sigma}=\left\|e^{-\sigma|x|} u\right\|_{E} \quad \text { and } \quad\left\|f_{i}\right\|_{i, \sigma}=\left\|e^{-\sigma|x|} f_{i}\right\|_{E_{i}} \quad(\sigma \geq 0)
$$

where $|x|=\sum_{j=1}^{n} x_{j}$. These norms fulfil condition (1.2) with $\psi(\sigma)=\exp (-\sigma|X|)$ and condition (2.2), respectively.

There holds $\left|m_{0}\right| \leq M_{0}$ with a positive constant $M_{0}$ and by (3.4) we have

$$
\begin{aligned}
e^{-\sigma|x|} & =\prod_{i=1}^{k} e^{-\sigma\left|\alpha_{i} x-\beta_{i} y\right|} \exp \left(-\sigma\left|\left(1-\sum_{i=1}^{k} \alpha_{i}\right) x\right|\right) \exp \left(-\sigma\left|\sum_{i=1}^{k} \beta_{i} y\right|\right) \\
& \leq \prod_{i=1}^{k} e^{-\sigma\left|\alpha_{i} x-\beta_{i} y\right|} .
\end{aligned}
$$

Hence using the Hölder inequality in view of (3.5), we obtain

$$
\begin{aligned}
& \left.\| K \mid f_{1}, \ldots, f_{k}\right) \|_{\sigma} \\
& \quad \leq M_{0} \underset{x \in D}{\operatorname{ess} \sup _{0}} \int_{0}^{x_{n}} \ldots \int_{0}^{x_{1}} \prod_{i=1}^{k}\left|m_{i}\left(\alpha_{i} x-\beta_{i} y\right) f_{i}\left(\alpha_{i} x-\beta_{i} y\right)\right| e^{-\sigma\left|\alpha_{i} x-\beta_{i} y\right|} d y_{1} \cdots d y_{n} \\
& \quad \leq C_{0} \prod_{i=1}^{k}\left(\int_{D}\left|m_{i}(z) f_{i}(z)\right|^{r_{i}} e^{-\sigma|z| r_{i}} d z_{1} \cdots d z_{n}\right)^{\frac{1}{r_{i}}}
\end{aligned}
$$

with

$$
C_{0}=M_{0} \prod_{i=1}^{k}\left(\prod_{j=1}^{n}\left|\beta_{i}^{j}\right|^{-1}\right)^{\frac{1}{r_{i}}} .
$$

Again by the Hölder inequality there hold the estimations

$$
\int_{D}\left|m_{i}(z) f_{i}(z)\right|^{r_{i}} e^{-\sigma|z| r_{i}} d z_{1} \cdots d z_{n} \leq M_{i}^{r_{i}}\left(\int_{D}\left|f_{i}(z)\right|^{p_{i}} e^{-\sigma|z| p_{i}} d z_{1} \cdots d z_{n}\right)^{\frac{r_{i}}{p_{i}}}
$$


with $M_{i}=\left(\int_{D}\left|m_{i}(z)\right|^{q_{i}} d z_{1} \cdots d z_{n}\right)^{\frac{1}{q_{i}}}$ and

$$
\int_{D}\left|m_{i}(z) f_{i}(z)\right|^{r_{i}} e^{-\sigma|z| r_{i}} d z_{1} \cdots d z_{n} \leq N_{i}^{r_{i}}(\sigma)\left(\int_{D}\left|f_{i}(z)\right|^{p_{i}} d z_{1} \cdots d z_{n}\right)^{\frac{r_{i}}{p_{i}}}
$$

with $N_{i}(\sigma)=\left(\int_{D}\left|m_{i}(z)\right|^{q_{i}} e^{-\sigma|z| q_{i}} d z_{1} \cdots d z_{n}\right)^{\frac{1}{q_{i}}}$ if $p_{i}<\infty$ (besides $q_{i}, r_{i}<\infty$ ). Therefore we have the desired inequalities

$$
\left\|K\left[f_{1}, \ldots, f_{k}\right]\right\|_{\sigma} \leq C \prod_{i=1}^{k}\left\|f_{i}\right\|_{i, \sigma}
$$

with $C=C_{0} \prod_{i=1}^{k} \cdot M_{i}$ and

$$
\left\|K\left[f_{1}, \ldots, f_{k}\right]\right\|_{\sigma} \leq \lambda(\sigma) \prod_{l \neq i}\left\|f_{l}\right\|_{l, \sigma} \cdot\left\|f_{i}\right\|_{i, 0} \quad(1 \leq i \leq k)
$$

with $\lambda(\sigma)=C_{0} \cdot \max _{i \in\{1, \ldots, k\}}\left(\prod_{l \neq i} M_{l} \cdot N_{i}(\sigma)\right)$ where by the Lebesgue dominant convergence theorem $N_{i}(\sigma) \rightarrow 0$ as $\sigma \rightarrow \infty$, hence also $\lambda(\sigma) \rightarrow 0$ as $\sigma \rightarrow \infty$. Corresponding inequalities hold in case $p_{i}=\infty$ for some $i \in\{1, \ldots, k\}$.

We point out the particular case $k=p+1(p \geq 1)$ with

$$
\alpha_{i}=0 \quad \text { and } \quad \beta_{i}=-\frac{1}{p} \quad(1 \leq i \leq p) \quad \text { and } \quad \alpha_{k}=\beta_{k}=1
$$

of (3.3) and (3.4), which leads for $f_{i}=u(1 \leq i \leq k)$ to the power operator of convolution type

$$
K_{p}[u]=\int_{0}^{x_{n}} \ldots \int_{0}^{x_{1}} m(x, y) u^{p}\left(\frac{y}{p}\right) u(x-y) d y_{1} \cdots d y_{n} .
$$

Examples of operators $G_{k, i}^{j}$ fulfilling the Lipschitz conditions (B3) with (B5) in the weighted norms (3.6) are also given by powers of functions with deviating argument, for instance. So let us consider in the space $E=C(\bar{D})$ or $E=L_{\infty}(D)$ the operator

$$
(G u)(x)=u^{p}(h(x)) \quad(p \geq 1, \text { entire })
$$

where $h \in C_{n}(\bar{D})$ is a continuous $n$-dimensional vector function satisfying $0 \leq h(x) \leq \frac{x}{p}$. We have

$$
\begin{aligned}
&\left|e^{-\sigma|x|}\left[\left(G u_{1}\right)(x)-\left(G u_{2}\right)(x)\right]\right| \\
&=\left|e^{-\sigma|x|}\left[u_{1}(h(x))-u_{2}(h(x))\right] \sum_{j=0}^{p-1} u_{1}^{j}(h(x)) u_{2}^{p-1-j}(h(x))\right| \\
& \leq e^{-\sigma|x-p h(x)|} e^{-\sigma|h(x)|}\left|u_{1}(h(x))-u_{2}(h(x))\right| \\
& \quad \times \sum_{j=0}^{p-1} e^{-\sigma j|h(x)|}\left|u_{1}^{j}(h(x))\right|: e^{-\sigma(p-1-j)|h(x)|}\left|u_{2}^{p-1-j}(h(x))\right| \\
& \leq\left\|u_{1}-u_{2}\right\|_{\sigma} \sum_{j=0}^{p-1}\left\|u_{1}\right\|_{\sigma}^{j}\left\|u_{2}\right\|_{\sigma}^{p-1-j}
\end{aligned}
$$


i.e. condition (B3) is fulfilled with $M\left(\rho_{1}, \rho_{2}\right)=\sum_{j=0}^{p-1} \rho_{1}^{j} \rho_{2}^{p-1-j}$.

As an application of the foregoing considerations we show that the initial-value problem for the one-dimensional integro-functional-differential equation

$$
\left.\begin{array}{rl}
v^{\prime}(t) & +\Phi\left(t, v(t), v^{2}\left(\frac{t}{2}\right), \ldots, v^{n_{0}}\left(\frac{t}{n_{0}}\right)\right) \\
& +\int_{0}^{t} a_{0}(s, t) v(t-s) d s+\int_{0}^{t} b_{0}(s, t) v^{\prime}(t-s) d s \\
& +\int_{0}^{t} v(t-s) \sum_{k=1}^{n_{1}} a_{k}(s, t) v^{k}\left(\frac{s}{k}\right) d s \\
& +\int_{0}^{t} v^{\prime}(t-s) \sum_{k=1}^{n_{2}} b_{k}(s, t) v^{k}\left(\frac{s}{k}\right) d s \\
& +\int_{0}^{t} v^{\prime}(t-s) \sum_{k=1}^{n_{3}} c_{k}(s, t)\left[v^{\prime}\left(\frac{s}{k}\right)\right]^{k} d s=g(t), \quad v(0)=c_{0}
\end{array}\right\}
$$

with $c_{0} \in \mathbb{R}$ and entire $n_{0}, n_{1}, n_{2}, n_{3} \geq 1$ has a unique solution $u \in C^{1}[0, T]$ for $g \in C[0, T]$ in any finite interval $[0, T](T>0)$, if the functions $a_{k}, b_{k}$ and $c_{k}$ are continuous and the function $\Phi\left(t, v_{1}, v_{2}, \ldots, v_{n_{0}}\right)$ is continuous and fulfills a uniform Lipschitz condition in the variables $\left(v_{1}, v_{2}, \ldots, v_{n_{0}}\right)$.

The statement immediately follows from the above results by taking $u=v^{\prime} \in C[0, T]$ with

$$
v(t)=\int_{0}^{t} u(s) d s+c_{0}
$$

as unknown function and observing that by the Young inequality

$$
\left\|v_{1}-v_{2}\right\|_{\sigma} \leq \min \left(T, \frac{1}{\sigma}\right)\left\|u_{1}-u_{2}\right\|_{\sigma}
$$

for the norm (3.6) in $C[0, T]$ (cp. [6: Example 5]). So the term with the function $\Phi$ generates an operator of the form $G_{0}$, the integral terms are operators of the form (3.1). Of course, the functions $v^{p}\left(\frac{t}{p}\right)$ in $(3.11)$ can be replaced by other operators of the form (3.10). For functional-differential equations of the form (3.11) without integrals cp. [3, 4], for instance.

We finally remark that further examples related to the examples in [6] are possible, also for systems of differential and integral equations and for operators $G_{k, i}^{j}$ with functional dependence on $u$. 


\section{References}

[1] Benedek, A. and R. Panzone: The spaces $L^{p}$. with mixed norm. Duke Math. J. 28 (1961), $301-324$.

[2] Bukhgeim, A. L.: Inverse problems of memory reconstruction. J. Inv. Ill-Posed Probl. 1 (1993), 193 - 205.

[3] El'sgol'z, L. E. and S. B. Norkin: Introduction in the Theory of Differential Equations with Deviated Argument (in Russian). Moscow: Nauka 1971.

[4] Hale, J. K.: Theory of Functional Differential Equations. New York: Springer-Verlag 1977.

[5] Wolfersdorf, L. von: A class of multi-dimensional nonlinear Volterra equations of convolution type. Demonstratio Math. 28 (1995), 807 - 820.

[6] Wolfersdorf, L. von and J. Janno: On a class of nonlinear convolution equations. Z. Anal. Anw. 14 (1995), 497 - 508.

Received 04.07.1996 